If you wish to distribute this article to others, you can order high-quality copies for your colleagues, clients, or customers by clicking here.

Permission to republish or repurpose articles or portions of articles can be obtained by following the guidelines here.

The following resources related to this article are available online at www.sciencemag.org (this infomation is current as of April 30, 2011):

Updated information and services, including high-resolution figures, can be found in the online version of this article at:

http://www.sciencemag.org/content/332/6029/577.full.html

Supporting Online Material can be found at:

http://www.sciencemag.org/content/suppl/2011/04/05/science.1201629.DC1.html

This article cites 22 articles, 1 of which can be accessed free:

http://www.sciencemag.org/content/332/6029/577.full.html\#ref-list-1

This article appears in the following subject collections:

Planetary Science

http://www.sciencemag.org/cgi/collection/planet_sci 
transform to the desired products $\mathbf{1 5}$ and $\mathbf{1 7}$. Additional silylium carborane and silane, plus extra hours of stirring, were required to achieve a higher yield. Treatment of substrate $\mathbf{1 8}$ afforded a yield of 51\%; the lower yield was probably caused by decomposition of the product (19).

The terphenyl substrates containing a methyl group on the nonfluorinated phenyl ring (10b and 12) showed a mixture of ortho and para products. The distribution was slightly in favor of the para position, which should be more accessible; however, the small preference for the para methyl indicates a very reactive intermediate that does not distinguish strongly between the two positions.

Additional studies enabled us to make a qualitative statement on the reaction rate. The terphenyl substrates $(\mathbf{8}, \mathbf{1 0 a}, \mathbf{1 0 b}$, and $\mathbf{1 2})$ were used in competitive $\mathrm{C}-\mathrm{F}$ activation reactions. Two substrates were added to the same reaction mixture with only half an equivalent of reagent. Gas chromatography-mass spectrometry (GCMS) consistently showed that substrates containing methyl groups (at any of the two rings) reacted faster than did the electron-poorer rings without substituents. The tendency of reaction rates for different substrates was found to be $\mathbf{8}>$ $\mathbf{1 0 a} \approx \mathbf{1 0 b}>12(25)$. This observation matches with the proposed transition state $\mathbf{A}$. Its positive charge would be stabilized by an increased electron density of the fluorophenyl ring, provided by an additional substituent such as the methyl group. Methylation of the donor arene had the same effect on the reaction rate.

For the transformation of substrate 2, quantum mechanical calculations predicted a transition state for the fluoride abstraction (Fig. 2) that nearby aryl moieties can stabilize $(18,25)$. The structural and activation parameters predicted by the quantum chemical model (calculated $E_{\mathrm{a}}=$ $19.8 \mathrm{kcal} \mathrm{mol}^{-1}$; transition-state interatomic lengths, $\mathrm{C}-\mathrm{F}=2.55 \AA, \mathrm{C}-\mathrm{C}=2.86 \AA$ ) fit well with the experimental findings (34). The model further supports the observation that a more electron-rich arene accelerates the reaction.

The proton is likely the universe's oldest catalyst, and it is now available as a crystalline arenium carborane (25). Coupled to a neutral silane as fuel, arenium acids are competent catalysts for the longsought phenyl cation-based Friedel-Crafts reactivity. Such C-F activation for the formation of arene-arene bonds complements transition metalbased arene-arene couplings, particularly for the formation of designed graphenes and higher-order polynuclear aromatic hydrocarbon-based materials. Understanding the science of silyl cations and phenyl cation-like intermediates at play here, along with the design of tailored precursors, will certainly lead to a useful expansion of the synthetic chemist's tool box and the material chemist's objects of investigation.

\section{References and Notes}

1. G. A. Olah, Friedel-Crafts Chemistry (Wiley, New York, 1973).

2. R. M. Roberts, A. A. Khalaf, Friedel-Crafts Alkylation Chemistry: A Century of Discovery (Dekker, New York, 1984).

3. H.-G. Franck, J. W. Stadelhofer, Industrial Aromatic Chemistry (Springer-Verlag, Berlin, 1988).

4. R. F. Heck, J. Am. Chem. Soc. 90, 5518 (1968).

5. Z. Rappoport, P. Stang, Eds., Dicoordinated Carbocations (Wiley, New York, 1997).

6. J. Hassan, M. Sévignon, C. Gozzi, E. Schulz, M. Lemaire, Chem. Rev. 102, 1359 (2002).

7. D. Alberico, M. E. Scott, M. Lautens, Chem. Rev. 107, 174 (2007).

8. N. Miyaura, A. Suzuki, Chem. Rev. 95, 2457 (1995).

9. H. Amii, K. Uneyama, Chem. Rev. 109, 2119 (2009)

10. V. P. W. Böhm, C. W. K. Gstottmayr, T. Weskamp, W. A. Herrmann, Angew. Chem. Int. Ed. 40, 3387 (2001).

11. The action of $\mathrm{SbF}_{5}$ on perfluorinated naphthalene forms radical cations capable of coupling with pentafluorinated benzene, albeit with limited substrate scope; see (35).

12. Flash vacuum pyrolysis appears to facilitate the lysis of $\mathrm{C}(\operatorname{aryl})-\mathrm{F}$ bonds and thereby promote arene-arene coupling; see (36).

13. C. Douvris, O. V. Ozerov, Science 321, 1188 (2008).

14. C. Douvris, C. M. Nagaraja, C. H. Chen, B. M. Foxman, O. v. Ozerov, J. Am. Chem. Soc. 132, 4946 (2010).
15. N. Lühmann, R. Panisch, T. Müller, Appl. Organomet. Chem. 24, 533 (2010).

16. S. J. Blanksby, G. B. Ellison, Acc. Chem. Res. 36, 255 (2003).

17. Y.-R. Luo, Comprehensive Handbook of Chemical Bond Energies (CRC Press, Boca Raton, FL, 2007).

18. S. Duttwyler et al., Angew. Chem. Int. Ed. 49, 7519 (2010).

19. C. A. Reed, Z. Xie, R. Bau, A. Benesi, Science 262, 402 (1993).

20. J. B. Lambert, W. J. Schulz, J. A. McConnell, W. Schilf, J. Am. Chem. Soc. 110, 2201 (1988).

21. C. Eaborn, J. Chem. Soc. (Resumed), 3148 (1953).

22. C. Eaborn, J. Chem. Soc. (Resumed), 4858 (1956).

23. C. Eaborn, J. Chem. Soc. 100, 43 (1975).

24. C. A. Reed, J. Chem. Soc. Chem. Commun. 2005, 1669 (2005).

25. See supporting material on Science Online.

26. J. B. Lambert, S. Zhang, S. M. Ciro, Organometallics $\mathbf{1 3}$ 2430 (1994).

27. S. Körbe, P. J. Schreiber, J. Michl, Chem. Rev. 106, 5208 (2006).

28. T. M. Douglas, E. Molinos, S. K. Brayshaw, A. S. Weller, Organometallics 26, 463 (2007).

29. C. A. Reed, Acc. Chem. Res. 31, 133 (1998).

30. M. Juhasz, S. Hoffmann, E. Stoyanov, K. C. Kim, C. A. Reed, Angew. Chem. Int. Ed. 43, 5352 (2004).

31. A. Nicolaides, D. M. Smith, F. Jensen, L. Radom, J. Am. Chem. Soc. 119, 8083 (1997).

32. R. Scholl, C. Seer, R. Weitzenbock, Chem. Ber. 43, 2202 (1910).

33. R. Scholl, C. Seer, Justus Liebigs Ann. Chem. 394, 111 (1912).

34. Specific coordination of solvent or counterion is estimated to have an effect of $<5 \mathrm{kcal} / \mathrm{mol}$ on activation parameters, such that the model is still consistent with a reaction at $80^{\circ} \mathrm{C}$ over several hours.

35. B. A. Selivanov, Y. V. Pozdnyakovich, T. V. Chuikova, O. I. Osina, V. D. Steingarts, Zh. Org. Khim. 16, 1910 (1980)

36. K. Y. Amsharov, M. A. Kabdulov, M. Jansen, Eur. J. Org. Chem. 2009, 6328 (2009)

Acknowledgments: We thank the Swiss National Science Foundation for financial support.

\section{Supporting Online Material}

www.sciencemag.org/cgi/content/full/332/6029/574/DC

Materials and Methods

Table $\$ 1$

References 37 to 45

3 January 2011; accepted 16 March 2011

10.1126/science.1202432

\title{
Venus's Southern Polar Vortex Reveals Precessing Circulation
}

\author{
D. Luz, ${ }^{1 *}$ D. L. Berry, ${ }^{2}$ G. Piccioni, ${ }^{3}$ P. Drossart, ${ }^{4}$ R. Politi, ${ }^{3}$ C. F. Wilson, ${ }^{5}$ S. Erard, ${ }^{4}$ F. Nuccilli ${ }^{6}$
}

Initial images of Venus's south pole by the Venus Express mission have shown the presence of a bright, highly variable vortex, similar to that at the planet's north pole. Using high-resolution infrared measurements of polar winds from the Venus Express Visible and Infrared Thermal Imaging Spectrometer (VIRTIS) instrument, we show the vortex to have a constantly varying internal structure, with a center of rotation displaced from the geographic south pole by $\sim 3$ degrees of latitude and that drifts around the pole with a period of 5 to 10 Earth days. This is indicative of a nonsymmetric and varying precession of the polar atmospheric circulation with respect to the planetary axis.

$\mathrm{V}$ enus has the most extreme atmospheric circulation of the terrestrial planets, with the cloud-level atmosphere spinning on average 60 times faster than the planet's surface (1). This superrotation $(2-10)$ extends to both polar regions in hemispheric spiral-like patterns of clouds (11), where it results in fast rotating, infrared-bright central vortices (12-15).

Although the spiral hemispheric patterns can be seen in ultraviolet dayside imagery, which re- veals upper cloud features at $\sim 70$ to $75 \mathrm{~km}$ altitude (11), thermal infrared observations reveal cloud structures at slightly lower altitudes of 65 to $70 \mathrm{~km}$ and show that the center of the southern polar vortex has a brightness temperature $\sim 15 \mathrm{~K}$ warmer than a surrounding cold collar (15). The mean cloud-top altitude is close to $74 \mathrm{~km}$ up to mid-latitudes in both hemispheres but decreases

${ }^{1}$ Centro de Astronomia e Astrofísica da Universidade de Lisboa, Observatório Astronómico de Lisboa, 1349-018 Lisboa, Portugal. 2Departamento de Física, Universidade de Évora, 7002-554 Évora, Portugal. ${ }^{3}$ Istituto Nazionale di Astrofisica, Istituto Nazionale di Astrofisica- Istituto di Astrofisica Spaziale e Fisica Cosmica (INAF-IASF) Rome, 00133 Rome, Italy. ${ }^{4}$ Laboratoire d'Études Spatiales et d'Instrumentation en Astrophysique, Observatoire de Paris, CNRS, Université Pierre et Marie Curie, Université Paris-Diderot, F-92195 Meudon Cedex, France. ${ }^{5}$ Department of Physics, University of Oxford, Oxford OX1 3PU, UK. ${ }^{6}$ INAF-Istituto di Fisica dello Spazio Interplanetario (IFSI) Rome, 00133 Rome, Italy.

*To whom correspondence should be addressed. E-mail: dluz@oal.ul.pt 
Fig. 1. (A) The S-shaped pattern of the southern polar vortex, frequently referred to as the polar dipole. Indicated is the apparent inversion of the zonal wind between the pole (cross) and the true center of rotation (white circle) if the latter is displaced from the planetary axis. The gray arrow indicates a zonal (eastwest) component that is locally opposite to the large-scale circulation (white arrow), showing that the centroid is displaced on average by $3^{\circ}$ from the geographic south pole. The image is the $5.0-\mu \mathrm{m}$ radiance map of the

A

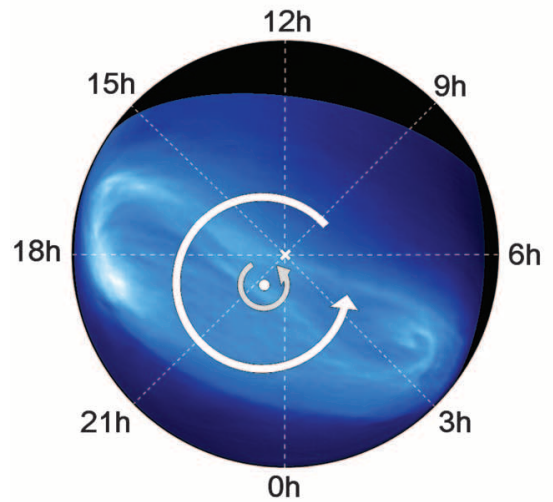

$B_{i}$
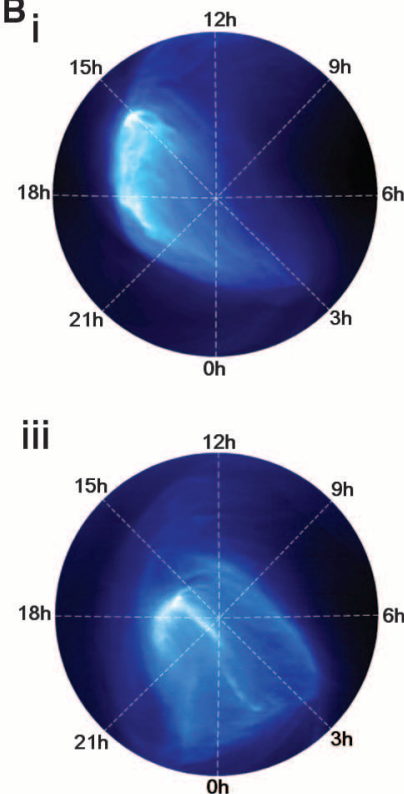

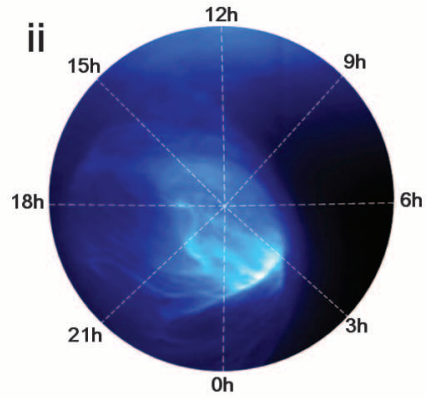

iv

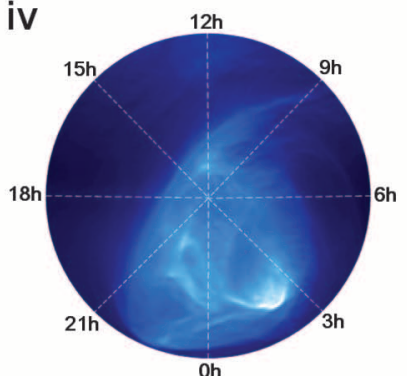

Fig. 2. Global mean (blue) zonal and (red) meridional wind components, obtained from feature tracking at $5.0 \mu \mathrm{m}$. Negative zonal wind indicates retrograde (westward) rotation.

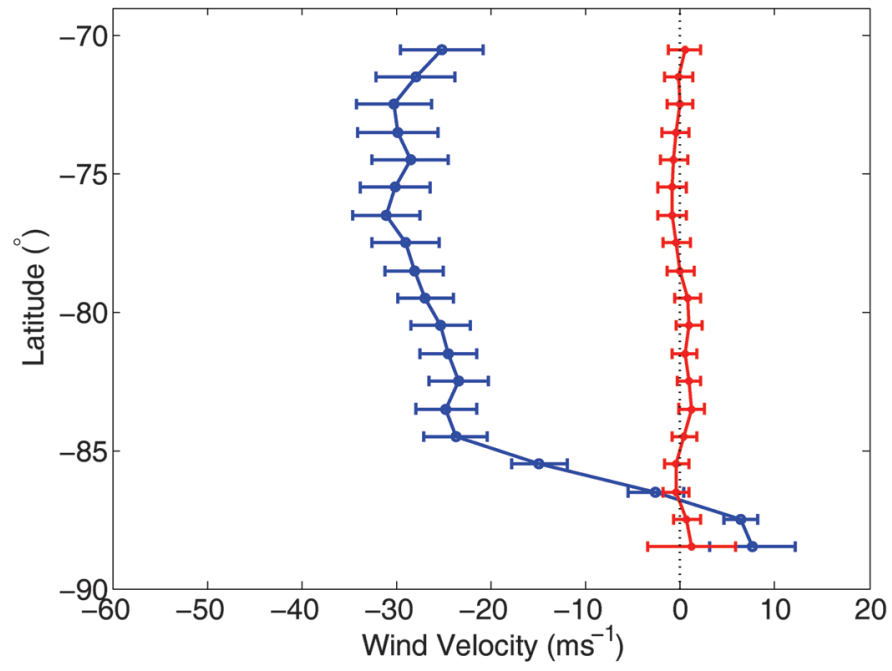

Fig. 3. Average positions of the centroids of the rotation in latitudelocal solar time coordinates, as measured from observations of the polar region at 3.8 and $5.0 \mu \mathrm{m}$, for a set of 34 orbits sampled from a period spanning 640 orbits. The colored points indicate sequential measurements made over short periods of up to 10 days (red, orbits 390, 392, 394, 396 and 398; green, orbits 473 to 479 ; black, orbits 668 , 672, 674, and 678; and purple, orbits 640,642, and 644); a cross indicates the first point in the sequence. The gray points indicate measurements that are not part of sufficiently long sequences. Superimposed points were displaced latitudinally by $0.25^{\circ}$ for clarity. The outer circle is the $80^{\circ} \mathrm{S}$ parallel.

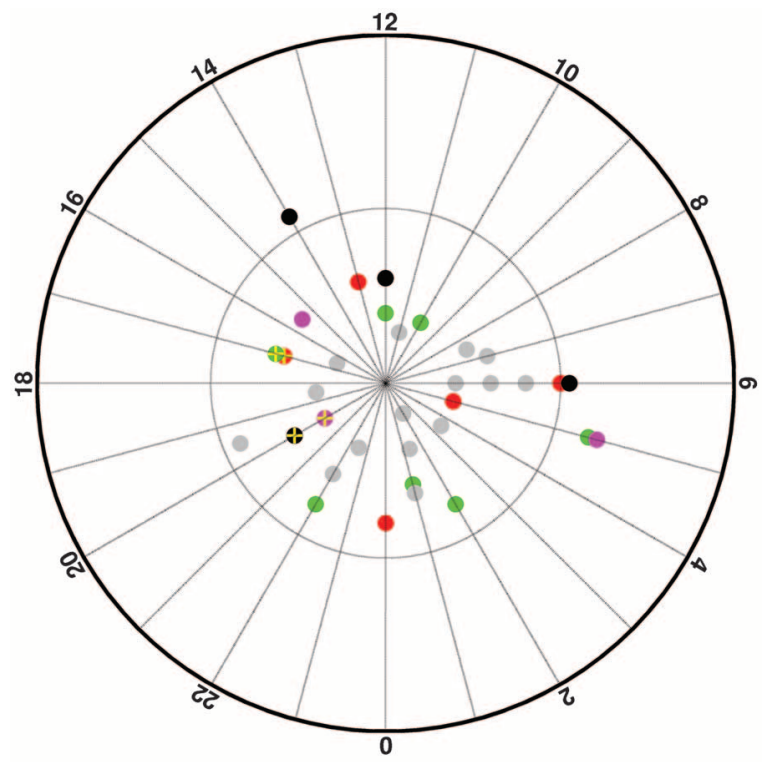

by 5 to $9 \mathrm{~km}$ at the pole and can experience fast variations (16). The decrease in cloud-top altitude, the increase in temperature, and the $\mathrm{CO}$ enrichment (17) at the center of the vortex may be indicative of downwelling in this region $(12,15)$.

The extended data set of Venus Express observations has shown the center of the southern polar vortex to be constantly changing in shape, on time scales of less than 24 hours. The longest imaging sequences obtained in a single orbit $(\sim 6$ hours) generally show a rotating stable structure, but its morphology when imaged in the next orbit ( 24 hours later) bears little resemblance with that of the previous day (Fig. 1).

Previous studies of the dynamical behavior of the Venus polar vortices were limited by the spatial or temporal resolution of the data $(14,15)$, in particular by the resolution of the infrared bright structure at the center, known as the polar dipole. Rotation rates measured for the northern dipole at $11.5 \mu \mathrm{m}$ (14) did not consider the fine structure of the vortex morphology, and the value obtained for its southern counterpart was based on a limited number of exposures. Although the northern polar vortex was occasionally seen to drift away from the pole (14), and this drifting was similarly observed once in its southern counterpart (15), this was not the subject of a detailed study.

Here, we present a study of the internal and global dynamics of the southern polar vortex from $70^{\circ} \mathrm{S}$ down to the south pole through use of high-spatial-resolution infrared images (at wavelengths of 3.8 and $5.0 \mu \mathrm{m}$ ) from Venus Express's Visible and Infrared Thermal Imaging Spectrometer (VIRTIS) (18). A particular advantage of using these wavelengths for polar-feature tracking is that they can be used simultaneously in both day- and night-side areas of the planet, probing the cloud layer at $\sim 65 \mathrm{~km}$ in the polar region (16). 
Fig. 4. (A) Monotonic local time coordinate of the centroid of rotation and linear fit for a set of four series of orbits lasting between 4 and 10 days (same color code as in Fig. 3). We used sequences of three orbits or more; time is in days counted from the start of the series (26). (B) Residues of the linear fits for the two best sampled sequences.

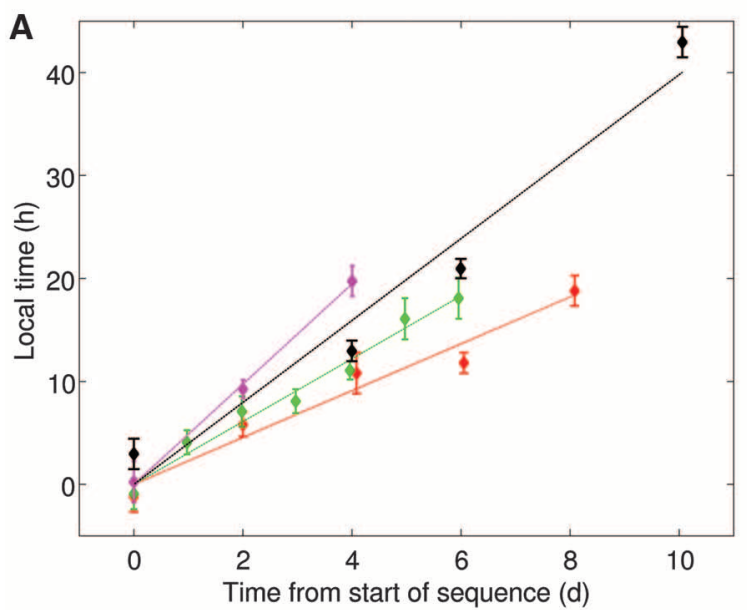

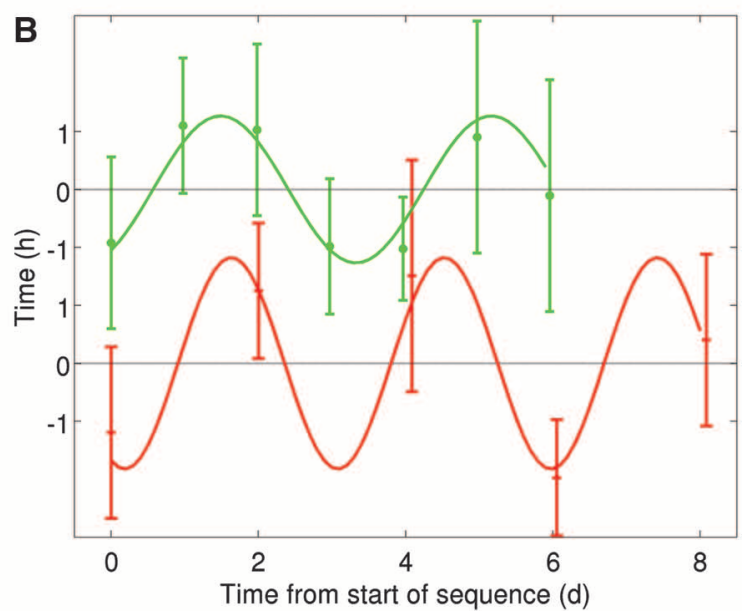

The latitudinal wind profile in this upper cloud layer indicates a mean zonal wind that is retrograde and approximately uniform equatorward of $84^{\circ} \mathrm{S}$, and a mean meridional wind that remains below the detection level (Fig. 2). The zonal component decreases strongly poleward of $84^{\circ} \mathrm{S}$ and changes sign to prograde close to $87^{\circ} \mathrm{S}$. A slightly slower wind is detected at $75^{\circ} \mathrm{S}$, coinciding with the poleward edge of the cold collar, which was previously identified as a region of colder air surrounding the bright center of the vortex (15). The inversion of the zonal wind close to the pole can be interpreted as a consequence of a nonalignment of the center of rotation of the infrared-bright feature with the planet's rotational axis. With such a displacement, an apparent inversion of the zonal wind between the pole and the true center of rotation results (Fig. 1A).

Clearly, the southern polar vortex is a morphologically variable, self-rotating, moving structure. It is also apparent that the position of its centroid of rotation is time variable over 24-hour periods and longer (Fig. 3). The majority of the centroids we measured are located poleward of $85^{\circ} \mathrm{S}$, with a near uniform spatial distribution. The mean latitude is $87^{\circ} \mathrm{S} \pm 1^{\circ}$, indicating that the center of polar vortex rotation is displaced from the south pole on average by $3^{\circ}$. These results, although admittedly from a small number of orbits, show no evidence of asymmetry of the positions of the centers of rotation with respect to local solar times, as might result from a solar tide-related forcing of the superrotation.

The centroid evolves around the pole in a precessional motion with near constant angular velocity (over time scales of up to a few days) (Fig. 4). Linear fits of the sequences yield motions of 2.3, 3.1, 4.0, and 4.9 hours/day in the local solar time frame ( 1 hour/day $=15 \%$ day $)$; the average motion is $\sim 52 \%$ day in the sense of the atmospheric rotation. This precessional motion is not uniform, however; it accelerates and decelerates (Fig. 4B). The residues of the linear fits can be fitted with sinusoids with periods of 2.5 days [orbits 390 to 398 (Fig. 4B, red)] and 3.6 days [orbits 473 to 479 (Fig. 4B, green)], indicating an oscillation with a period close to 3 days.

Lastly, the morphological variation of the vortex center can be shown to be the result of differential rotation. We have computed the rotation rates about the centroid of rotation from the average zonal wind component obtained in each orbit. Mean rotation rates were computed for three different regions: the inner core within $5^{\circ}$ of the centroid, the outer annulus also $5^{\circ}$ wide, and the extended core (the inner core plus the annulus), $10^{\circ}$ in radius. At cloud-top altitude, a $5^{\circ}$ angular distance corresponds to $534 \mathrm{~km}$ along the meridians. Using this method, we measured periods of $2.3 \pm 1.9$ days for the inner core, $2.8 \pm$ 1.9 days for the outer annulus, and $2.6 \pm 1.9$ days for the extended core region [compared with the rotation rate of $2.48 \pm 0.05$ days obtained previously (15)].

These results show that the southern polar atmospheric circulation is nonaligned with, and precesses about, the rotational axis of the planet. This may indicate that the region of maximum downwelling at the polar vortices - which is intimately linked with the general circulation of the atmosphere - is not static over the pole but drifts around it. In current theory, the GieraschRossow-Williams mechanism for the origin of Venus's superrotation $(19,20)$ implies a poleward transport of momentum by the mean meridional circulation in the upper branch of the Hadley cells (which on Venus extend from the equator to the poles), balanced by equatorward transport by eddies in the mid-latitudes. Non-axisymmetric eddy motions are required because axially symmetric advective processes cannot account for the transfer of angular momentum from high to low latitudes (21). The nonalignment of the polar vortex with the planetary axis makes the polar circulation naturally non-axisymmetric and therefore capable of effecting this transport of momentum, as suggested previously (19). Moreover, because polar vortices are driven by the thermal contrast between the equator and the poles via the Hadley cells (22) the nonalignment and drift of the polar vortex may be an indication that the region of subsidence in the descending branch of the Hadley cell is also nonaligned and drifting.

Polar vortices have interesting dynamical behavior, as has been shown in atmospheres throughout the solar system $(23,24)$. Our results highlight the importance of the polar vortex in the global dynamics of the Venus atmosphere, a phenomenon still not taken into account in current models of global circulation, which cannot simultaneously capture the dynamics of the vortices and superrotation.

\section{References and Notes}

1. P. Gierasch et al., in Venus II: Geology, Geophysics, Atmosphere, and Solar Wind Environment,

S. W. Bougher, D. M. Hunten, R. J. Philips, Eds. (Arizona Univ. Press, Tucson, AZ, 1997).

2. M. Y. Marov, Annu. Rev. Astron. Astrophys. 16, 141 (1978).

3. G. Schubert, in Venus (Arizona Univ. Press, Tucson, AZ, 1983)

4. S. Limaye, V. E. Suomi, ]. Atmos. Sci. 38, 1220 (1981).

5. W. B. Rossow, A. D. del Genio, T. Eichler, J. Atmos. Sci. 47, 2053 (1990).

6. A. Toigo, P. J. Gierasch, M. D. Smith, Icarus 109, 318 (1994)

7. J. Peralta, R. Hueso, A. Sánchez-Lavega, Icarus 190, 469 (2007).

8. W. J. Markiewicz et al., Nature 450, 633 (2007).

9. R. Moissl et al., J. Geophys. Res. Planets 114, E00B31 (2009).

10. A. Sánchez-Lavega et al., Geophys. Res. Lett. 35, L13204 (2008)

11. V. E. Suomi, S. S. Limaye, Science 201, 1009 (1978).

12. F. W. Taylor, D. J. McCleese, D. ]. Diner, Nature 279, 613 (1979).

13. F. W. Taylor et al., J. Geophys. Res. 85 (A13), 7963 (1980).

14. J. T. Schofield, D. J. Diner, Nature 305, 116 (1983).

15. G. Piccioni et al., Nature 450, 637 (2007).

16. N. Ignatiev et al., J. Geophys. Res. Planets 114, E00B43 (2009).

17. P. Irwin et al., J. Geophys. Res. Planets 113, E00B01 (2008).

18. Materials and methods are available as supporting material on Science Online.

19. P. Gierasch, ]. Atmos. Sci. 32, 1038 (1975).

20. W. B. Rossow, G. P. Williams, J. Atmos. Sci. 36, 377 (1979).

21. R. Hide, J. Atmos. Sci. 26, 841 (1969).

22. S. Limaye, J. Geophys. Res. 112 (E4), E04S09 (2007).

23. S. Limaye et al., Geophys. Res. Lett. 36, L04204 (2009).

24. U. Dyudina et al., Icarus 202, 240 (2009).

25. The VIRTIS mapping infrared channel provided a large data set from the Venus orbit insertion in April 2006; 
however, only a fraction of image sequences with exposure times optimized for observation at $5.0 \mu \mathrm{m}$ were obtained at high spatial resolution for the polar region.

26. The local time coordinate, which is cyclical, has been linearized by adding 24 hours whenever a cycle was completed (for example, the sequence $[-5,10,-1,-8]$ turns into $[-5,10,23,40])$.

Acknowledgments: D.L. and D.L.B. acknowledge support from Fundação para a Ciência e a Tecnologia project
PTDC/CTE-AST/110702/2009, and C.F.W. from the UK's Science and Technology Facilities Council and from the European Space Agency (ESA). The authors thank ESA, Agenzia Spaziale Italiana, Centre National d'Études Spatiales, and the other national agencies for supporting the Venus Express mission and acknowledge support from the VIRTIS/VEX technical team. They are also grateful to three anonymous referees for constructive comments.

\section{Supporting Online Material}

www.sciencemag.org/cgi/content/full/science.1201629/DC1 Materials and Methods

Reference 27

13 December 2010; accepted 25 March 2011

Published online 7 April 2011;

10.1126/science.1201629

\section{Surface-Generated Mesoscale Eddies Transport Deep-Sea Products from Hydrothermal Vents}

\begin{abstract}
Diane K. Adams, ${ }^{1 *} \dagger$ Dennis ]. McGillicuddy Jr., ${ }^{1}$ Luis Zamudio, ${ }^{2}$ Andreas M. Thurnherr, ${ }^{3}$ Xinfeng Liang, ${ }^{3}$ Olivier Rouxel, ${ }^{1,4}$ Christopher R. German, ${ }^{1}$ Lauren S. Mullineaux ${ }^{1}$
\end{abstract}

Atmospheric forcing, which is known to have a strong influence on surface ocean dynamics and production, is typically not considered in studies of the deep sea. Our observations and models demonstrate an unexpected influence of surface-generated mesoscale eddies in the transport of hydrothermal vent efflux and of vent larvae away from the northern East Pacific Rise. Transport by these deep-reaching eddies provides a mechanism for spreading the hydrothermal chemical and heat flux into the deep-ocean interior and for dispersing propagules hundreds of kilometers between isolated and ephemeral communities. Because the eddies interacting with the East Pacific Rise are formed seasonally and are sensitive to phenomena such as El Niño, they have the potential to introduce seasonal to interannual atmospheric variations into the deep sea.

$\mathrm{H}$ ydrothermal vents are hot spots for geological, geochemical, and biological activity that alter the global oceanic heat and chemical budgets $(1,2)$ and support unique chemosynthetic communities $(3,4)$. The disjunct distribution and transient nature of vents pose challenges for exporting vent-derived heat and chemicals into the global ocean and transporting propagules between distant vent fields. On short time scales, vent-derived products can remain close to the ridge axis. Chemical tracers can be used to locate hydrothermal vents (5), and larval supply to established vents is predominantly from local sources $(6,7)$. However, larvae and much of the seawater chemically altered at vents are eventually transported to distant locales. Hydrothermal seawater alterations contribute to the removal and addition of major chemical constituents (1), such as $\mathrm{Ca}$ and $\mathrm{Mg}$, as well as trace metals, such as $\mathrm{Fe}(8,9)$ which may affect local and global biogeochemical cycling (10-12). Vent larvae must, at least episodically, undergo long-distance dispersal to maintain observations of

${ }^{1}$ Woods Hole Oceanographic Institution, Woods Hole, MA 02543, USA. ${ }^{2}$ Center for Ocean-Atmospheric Prediction Studies, Florida State University, Tallahassee, FL 32306, USA. ${ }^{3}$ LamontDoherty Earth Observatory, Palisades, NY 10964, USA. ${ }^{4}$ Université Européenne de Bretagne, Université de Brest, Institut Universaire Européen de la Mer (IUEM), UMR 6538, Insititut Français de Recherche pour l'Exploitation de la Mer (IFREMER), BP 80 F- 29280 Plouzané, France.

*Present address: National Institutes of Health, National Institute of Dental and Craniofacial Research, Bethesda, MD 20892, USA.

†To whom correspondence should be addressed. E-mail: dadams@whoi.edu high gene flow $(13,14)$ and rapid colonization of disturbed and nascent vents $(7,15)$. Ridge-trapped jets $(16,17)$ and hydrothermally induced flows (18) have the potential to transport material near the ridge. Yet after decades of research at hydrothermal vents, the mechanisms by which heat, chemicals, and larvae are transported throughout the global ocean and between distant vent fields remain poorly resolved.

To investigate mechanisms that transport vent fluids and larvae, we performed time-series observations of hydrodynamics and larval, chemi$\mathrm{cal}$, and mass fluxes in the $9^{\circ} 50^{\prime} \mathrm{N}$ area of the

Fig. 1. Observations at the EPR ridge crest, East Wall. (A) Supply of vent gastropod larvae and (B) mass flux. Solid line indicates the mean flux. Dashed lines indicate the $95 \%$ confidence interval (Cl). (C) Current velocities recorded at $170 \mathrm{~m}$ above bottom (2350 m depth). Shown are the strong currents and rapid changes in direction in March 2005. (D) Variability of Fe flux over the time series. Solid bars indicate samples analyzed as before (pre-) the current anomaly. Open bars indicate samples during or after (post-) the current anomaly.
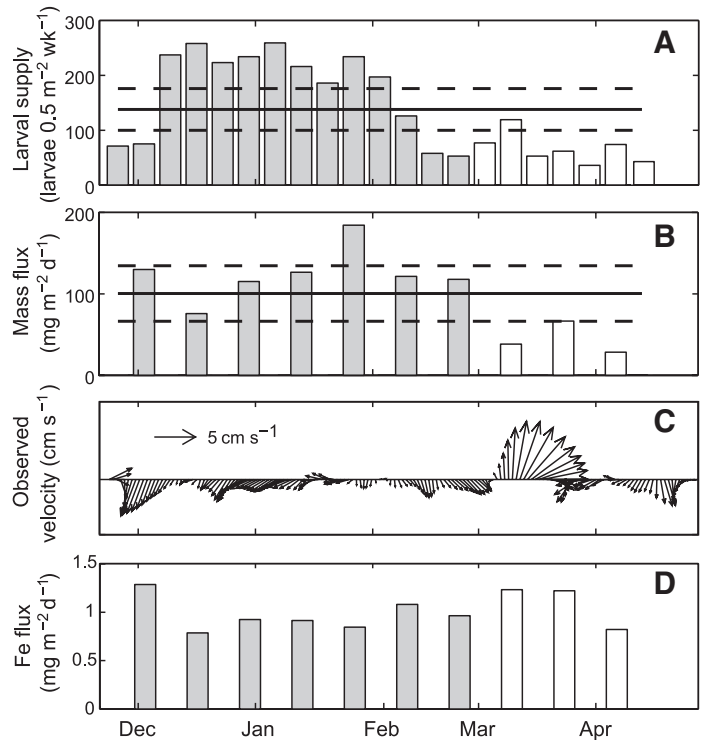

East Pacific Rise (EPR) (fig. S1). Dramatic decreases in the larval supply of vent gastropods (Fig. 1A and figs. S2 and S3) and in mass flux associated with settling particles (Fig. 1B) were observed near the end of the time series and corresponded to a period of anomalous current velocities. Current velocities both on-axis (Fig. 1C and fig. S4, A and B) and off-axis (fig. S4C) reached speeds exceeding $15 \mathrm{~cm} \mathrm{~s}^{-1}$ during the anomaly, compared with the mean speed of $5.5 \mathrm{~cm} \mathrm{~s}^{-1}$ (at $170 \mathrm{~m}$ above bottom). Using a permutation test, larval and mass fluxes were significantly lower than expected post-anomaly (during and after the velocity anomaly): $P=0.0007$ and $P=0.008$, respectively. The biological and geochemical changes appear to have been driven by anomalous low-frequency currents (Fig. 1C) because the magnitude of high-frequency motions was relatively stable during this time (fig. S5).

The concurrent decreases (19) in larval supply and mass flux were most likely due to hydrodynamic transport away from the ridge rather than changes in source production. Both larval supply and mass flux are derived from independent pools built up over time, so changes in production would be observed as dampened or lagged changes in flux. Additionally, it is unlikely that mass flux sources and multiple species' reproduction changed concurrently. Hydrothermal vent gastropods typically exhibit continuous or quasi-continuous reproduction $(20,21)$ with pre-competency periods. Therefore, we assume our samples come from a continuously produced larval pool that integrates reproductive output over time. 